\title{
Convergence Behavior for Newton-Steffensen's Method under $\gamma$-Condition of Second Derivative
}

\author{
Yonghui Ling, ${ }^{1} \mathrm{Xiubin} \mathrm{Xu}^{2}$ and Shaohua $\mathrm{Yu}^{2}$ \\ ${ }^{1}$ Department of Mathematics, Zhejiang University, Hangzhou 310027, China \\ ${ }^{2}$ Department of Mathematics, Zhejiang Normal University, Jinhua 321004, China \\ Correspondence should be addressed to Xiubin Xu; xxu@zjnu.edu.cn
}

Received 2 September 2013; Accepted 30 September 2013

Academic Editor: Jen-Chih Yao

Copyright (C) 2013 Yonghui Ling et al. This is an open access article distributed under the Creative Commons Attribution License, which permits unrestricted use, distribution, and reproduction in any medium, provided the original work is properly cited.

The present paper is concerned with the semilocal as well as the local convergence problems of Newton-Steffensen's method to solve nonlinear operator equations in Banach spaces. Under the assumption that the second derivative of the operator satisfies $\gamma$-condition, the convergence criterion and convergence ball for Newton-Steffensen's method are established.

\section{Introduction}

Let $X$ and $Y$ be real or complex Banach spaces, let $D \subset X$ be an open subset, and let $F: D \subset X \rightarrow Y$ be the Fréchet differentiable nonlinear operator. Approximating a solution of a nonlinear equation

$$
F(x)=0
$$

is widely studied in both theoretical and applied areas of mathematics.

One of the most famous methods to solve this problem is Newton's method defined by

$$
x_{n+1}=x_{n}-F^{\prime}\left(x_{n}\right)^{-1} F\left(x_{n}\right), \quad n=0,1,2, \ldots,
$$

where $x_{0} \in D$ is an initial point. Usually, the study about convergence issue of Newton's method includes local and semilocal convergence analyses. The local convergence issue is, based on the information around a solution, to seek estimates of the radii of convergence balls, while the semilocal one is, based on the information around an initial point, to give criteria ensuring the convergence. Among the semilocal convergence results on Newton's method, one of the famous results is Smale's point estimate theory which gives a convergence criterion of Newton's method only based on the information at the initial point for analytic functions; see for example, [1-6]. To extend and improve Smale's theory,
Wang and Han proposed in $[7,8]$ the notion of $\gamma$-condition, which is weaker than Smale's assumption in [5] for analytic operators.

There are several kinds of cubic generalizations for Newton's method. The most important family is the EulerHalley family and its variations which include Chebyshev's method and Halley's method as special cases; see for example, [9-16] and references therein. However, the disadvantage of this family is that the evaluation of the second derivative of the operator $F$ is required at every step, the operation cost of which may be very large in fact. To reduce the operation cost but also retain the cubic convergence, Sharma in [17] proposed the following Newton-Steffensen's method which avoids the computation of the second Fréchet derivative. Let $f: \mathbb{R} \rightarrow \mathbb{R}$. The method is defined as follows:

$$
\begin{gathered}
y_{n}=x_{n}-\frac{f\left(x_{n}\right)}{f^{\prime}\left(x_{n}\right)}, \\
x_{n+1}=x_{n}-\frac{f\left(x_{n}\right)}{g\left(x_{n}\right)}, \quad n=0,1, \ldots,
\end{gathered}
$$

where $g\left(x_{n}\right)=\left(\left(f\left(y_{n}\right)-f\left(x_{n}\right)\right) /\left(y_{n}-x_{n}\right)\right)$. The author obtained cubic convergence for (3) under the assumption that $f$ is sufficiently smooth in the neighborhood of the solution. 
Motivated by the work mentioned above, we extend this method to Banach spaces and present its semilocal and local convergence. The extension is described as follows:

$$
\begin{gathered}
y_{n}=x_{n}-F^{\prime}\left(x_{n}\right)^{-1} F\left(x_{n}\right), \\
x_{n+1}=x_{n}-\left[y_{n}, x_{n} ; F\right]^{-1} F\left(x_{n}\right), \quad n=0,1, \ldots,
\end{gathered}
$$

where the divided difference operator is defined by

$$
\left[y_{n}, x_{n} ; F\right]=\int_{0}^{1} F^{\prime}\left(x_{n}+t\left(y_{n}-x_{n}\right)\right) \mathrm{d} t .
$$

In Section 2, we introduce some preliminary notions and important majorizing functions with properties. In Sections 3 and 4, we study the semilocal convergence and local convergence results of Newton-Steffensen's method under $\gamma$ condition, respectively. We obtain the uniqueness ball and the convergence ball.

\section{Notations and Preliminary Results}

Throughout this paper, we assume that $X$ and $Y$ are two Banach spaces. Let $D \subset X$ be an open subset and let $F: \mathrm{D} \subset X \rightarrow Y$ be a nonlinear operator with the continuous twice Fréchet derivative. For $x \in X$ and $r>$ 0 , we use $\mathbf{B}(x, r)$ and $\overline{\mathbf{B}(x, r)}$ to denote the open ball with radius $r$ and center $x$ and its closure, respectively. Let $\bar{x} \in$ $D$ be such that $F^{\prime}(\bar{x})^{-1}$ exists and $\overline{\mathbf{B}(\bar{x}, r)} \subset D$.

Let $\gamma$ be some positive constant and $0<r \leqslant(1 / \gamma)$. We say that $F$ satisfies $\gamma$-condition on $\mathbf{B}(\bar{x}, r)$ if the following relation holds:

$$
\left\|F^{\prime}(\bar{x})^{-1} F^{\prime \prime}(x)\right\| \leqslant \frac{2 \gamma}{(1-\gamma\|x-\bar{x}\|)^{3}}, \quad x \in \mathbf{B}(\bar{x}, r) .
$$

For simplicity, we write

$$
r_{0}=\left(1-\frac{1}{\sqrt{2}}\right) \frac{1}{\gamma}
$$

The lemma below is useful in the next two sections.

Lemma 1. Suppose that $r \leqslant r_{0}$ and that $F$ satisfies $\gamma$ condition (6) on $\mathbf{B}(\bar{x}, r)$. Then for any $x \in$ $\mathbf{B}(\bar{x}, r), F^{\prime}(x)^{-1}$ exists and the following inequality holds:

$$
\left\|F^{\prime}(x)^{-1} F^{\prime}(\bar{x})\right\| \leqslant \frac{(1-\gamma\|x-\bar{x}\|)^{2}}{2(1-\gamma\|x-\bar{x}\|)^{2}-1} .
$$

Proof. We can derive the following relation:

$$
\begin{aligned}
I-F^{\prime} & (\bar{x})^{-1} F^{\prime}(x) \\
& =-\int_{0}^{1} F^{\prime}(\bar{x})^{-1} F^{\prime \prime}(\bar{x}+t(x-\bar{x}))(x-\bar{x}) \mathrm{d} t .
\end{aligned}
$$

For any $x \in B(\bar{x}, r)$, it follows from $\gamma$-condition and $r \leqslant$ $r_{0}$ that

$$
\begin{aligned}
\left\|I-F^{\prime}(\bar{x})^{-1} F^{\prime}(x)\right\| & \leqslant \int_{0}^{1} \frac{2 \gamma\|x-\bar{x}\|}{(1-\gamma\|x-\bar{x}\| t)^{3}} \mathrm{~d} t \\
& =\frac{1}{(1-\gamma\|x-\bar{x}\|)^{2}}-1<1 .
\end{aligned}
$$

Then, by Banach lemma, one has that $F^{\prime}(x)^{-1}$ exists and the following inequality holds:

$$
\begin{aligned}
\left\|F^{\prime}(x)^{-1} F^{\prime}(\bar{x})\right\| & \leqslant \frac{1}{1-\left[\left(1 /(1-\gamma\|x-\bar{x}\|)^{2}\right)-1\right]} \\
& =\frac{(1-\gamma\|x-\bar{x}\|)^{2}}{2(1-\gamma\|x-\bar{x}\|)^{2}-1} .
\end{aligned}
$$

Let $\beta>0$ be some positive constant. The following majorizing function $h$ introduced by Wang and Han in [18] will be used to obtain a Smale-type semilocal convergence criterion:

$$
h(t)=\beta-t+\frac{\gamma t^{2}}{1-\gamma t}, \quad t \in\left[0, \frac{1}{\gamma}\right) .
$$

Let $\left\{s_{n}\right\}$ and $\left\{t_{n}\right\}$ denote the corresponding sequences generated by Newton-Steffensen's method for the majorizing function $h$ with the initial point $t_{0}=0$; that is,

$$
\begin{gathered}
s_{n}=t_{n}-\frac{h\left(t_{n}\right)}{h^{\prime}\left(t_{n}\right)} \\
t_{n+1}=t_{n}-\left(\frac{h\left(s_{n}\right)-h\left(t_{n}\right)}{s_{n}-t_{n}}\right)^{-1} h\left(t_{n}\right), \quad n=0,1, \ldots
\end{gathered}
$$

The following lemma taken from [19] describes some useful properties about $h$.

Lemma 2. Suppose that

$$
\alpha:=\beta \gamma \leqslant 3-2 \sqrt{2}
$$

Then $h$ has two zeros in $[0,1 / \gamma)$ denoted by $t^{*}$ and $t^{* *}$. They satisfy the following relations:

$$
\beta \leqslant t^{*} \leqslant r_{0} \leqslant t^{* *} \leqslant \frac{1}{2 \gamma} .
$$

Moreover, $h$ is decreasing monotonically in interval $\left[0, r_{0}\right]$, while it is increasing monotonically in interval $\left[r_{0}, 1 /(2 \gamma)\right]$.

The lemma below describes the convergence property of the sequences $\left\{s_{n}\right\}$ and $\left\{t_{n}\right\}$, which is crucial for the semilocal convergence analysis of Newton-Steffensen's method (4) under $\gamma$-condition. 
Lemma 3. Suppose that (14) holds. Let $\left\{s_{n}\right\}$ and $\left\{t_{n}\right\}$ be the sequences generated by (13). Then

$$
0 \leqslant t_{n}<s_{n}<t_{n+1}<t^{*} \quad \forall n \geqslant 0 .
$$

Moreover, $\left\{s_{n}\right\}$ and $\left\{t_{n}\right\}$ converge increasingly to the same point $t^{*}$.

Proof. To show that (16) holds for $n=0$, we note that $0=$ $t_{0}<s_{0}=\beta$ and that $t_{1}=(\beta(1-\gamma \beta) /(1-2 \gamma \beta))$. By (15), we have

$$
\frac{1-\gamma \beta}{1-2 \gamma \beta}>1
$$

This implies that $t_{1}>\beta=s_{0}$. It remains to show that $t_{1}<$ $t^{*}$ for the case $n=0$. To this end, we define a real function as

$$
\Phi(t)=1-\frac{\gamma t}{1-\gamma t}, \quad t \in[0,+\infty)
$$

It is clear that $\Phi(t)=-(h(t)-\beta) / t$ and that $\Phi(t)$ is decreasing monotonically in $[0,+\infty)$. It follows from (15) that $\Phi(\beta)>\Phi\left(t^{*}\right)$. In view of the fact that $t^{*}$ is the unique zero of $h$ in $\left[0, r_{0}\right]$, we obtain $\beta / t^{*}=\Phi\left(t^{*}\right)<\Phi(\beta)$. This is equivalent to

$$
t^{*}>\frac{\beta}{1-(\gamma \beta /(1-\gamma \beta))}=t_{1}
$$

Hence (16) holds for $n=0$.

Now we assume that

$$
0 \leqslant t_{n-1}<s_{n-1}<t_{n}<t^{*} \text { for some } n \geqslant 1 \text {. }
$$

From Lemma 2, we have $h(t) \geqslant 0$, for each $t \in\left[0, t^{*}\right]$, and $h\left(t_{n}\right) / h^{\prime}\left(t_{n}\right)<0$. The later one implies that $s_{n}>t_{n}$. Define function

$$
N(t)=t-\frac{h(t)}{h^{\prime}(t)}, \quad t \in\left[0, t^{*}\right] .
$$

Then, $N^{\prime}(t)=h(t) h^{\prime \prime}(t) / h^{\prime}(t)^{2}>0$, which implies that $N$ is increasing monotonically in $\left[0, t^{*}\right]$. Hence, we have

$$
s_{n}=t_{n}-\frac{h\left(t_{n}\right)}{h^{\prime}\left(t_{n}\right)}<t^{*}-\frac{h\left(t^{*}\right)}{h^{\prime}\left(t^{*}\right)}=t^{*} .
$$

Since $h$ is convex in $\left[0, t^{*}\right]$, we get $h^{\prime}\left(t_{n}\right)<\left(h\left(s_{n}\right)-h\left(t_{n}\right)\right) /$ $\left(s_{n}-t_{n}\right)$ and so $s_{n}<t_{n+1}$.

Furthermore, we claim that

$$
\begin{aligned}
& t^{\prime}-\left(\frac{h(t)-h\left(t^{\prime}\right)}{t-t^{\prime}}\right)^{-1} h\left(t^{\prime}\right) \\
& \quad<t^{\prime \prime}-\left(\frac{h\left(t^{\prime \prime}\right)-h(t)}{t^{\prime \prime}-t}\right)^{-1} h\left(t^{\prime \prime}\right)
\end{aligned}
$$

for all $t^{\prime}, t, t^{\prime \prime} \in\left[0, t^{*}\right]$ and $t^{\prime}<t<t^{\prime \prime}$. Indeed, it follows from the convexity of $h$ that

$$
\begin{aligned}
-\frac{h\left(t^{\prime \prime}\right)}{h^{\prime}(t)} & \leqslant-\left(\frac{h\left(t^{\prime \prime}\right)-h(t)}{t^{\prime \prime}-t}\right)^{-1} h\left(t^{\prime \prime}\right) \\
& \leqslant-\frac{h\left(t^{\prime \prime}\right)}{h^{\prime}\left(t^{\prime \prime}\right)}, \\
-\frac{h\left(t^{\prime}\right)}{h^{\prime}\left(t^{\prime}\right)} & \leqslant-\left(\frac{h(t)-h\left(t^{\prime}\right)}{t-t^{\prime}}\right)^{-1} h\left(t^{\prime}\right) \\
& \leqslant-\frac{h\left(t^{\prime}\right)}{h^{\prime}(t)},
\end{aligned}
$$

from which we have

$$
\begin{aligned}
\left(t^{\prime \prime}-t^{\prime}\right)+\frac{h\left(t^{\prime}\right)-h\left(t^{\prime \prime}\right)}{h^{\prime}(t)} & \leqslant\left(t^{\prime \prime}-t^{\prime}\right)+\left(T^{\prime \prime}-T^{\prime}\right) \\
& \leqslant\left(t^{\prime \prime}-t^{\prime}\right)-\frac{h\left(t^{\prime \prime}\right)}{h^{\prime}\left(t^{\prime \prime}\right)}+\frac{h\left(t^{\prime}\right)}{h^{\prime}\left(t^{\prime}\right)},
\end{aligned}
$$

where

$$
\begin{aligned}
& T^{\prime}=-\left(\frac{h(t)-h\left(t^{\prime}\right)}{t-t^{\prime}}\right)^{-1} h\left(t^{\prime}\right), \\
& T^{\prime \prime}=-\left(\frac{h\left(t^{\prime \prime}\right)-h(t)}{t^{\prime \prime}-t}\right)^{-1} h\left(t^{\prime \prime}\right) .
\end{aligned}
$$

Noting that $-1<h^{\prime}(t)<0$ for all $t \in\left[0, t^{*}\right]$, we obtain

$$
\left(t^{\prime \prime}-t^{\prime}\right)+\frac{h\left(t^{\prime}\right)-h\left(t^{\prime \prime}\right)}{h^{\prime}(t)}>\left(t^{\prime \prime}-t^{\prime}\right)+\left[h\left(t^{\prime \prime}\right)-h\left(t^{\prime}\right)\right]
$$

$\geqslant 0$

Then (23) follows. By (23), we conclude that

$$
\begin{aligned}
t_{n+1} & =t_{n}-\left(\frac{h\left(s_{n}\right)-h\left(t_{n}\right)}{s_{n}-t_{n}}\right)^{-1} h\left(t_{n}\right) \\
& <t^{*}-\left(\frac{h\left(t^{*}\right)-h\left(s_{n}\right)}{t^{*}-s_{n}}\right)^{-1} h\left(t^{*}\right)=t^{*} .
\end{aligned}
$$

Therefore, (16) holds for all $n \geqslant 0$. The inequlities in (16) imply that $\left\{s_{n}\right\}$ and $\left\{t_{n}\right\}$ converge increasingly to some same points, say $\tau$. Clearly $\tau \in\left[0, t^{*}\right]$ and $\tau$ is a zero of $h$ in $\left[0, t^{*}\right]$. Noting that $t^{*}$ is the unique zero of $h$ in $\left[0, r_{0}\right]$, one has that $\tau=t^{*}$. The proof is complete. 


\section{Convergence Criterion}

Throughout this subsection, let $x_{0} \in D$ be the initial point such that the inverse $F^{\prime}\left(x_{0}\right)^{-1}$ exists and let $\mathbf{B}\left(x_{0}, r_{0}\right) \subset D$, where $r_{0}$ is defined by (7). Moreover, we assume that $F$ satisfies $\gamma$-condition on $\mathbf{B}\left(x_{0}, r_{0}\right)$; that is, the following relation holds:

$$
\begin{array}{r}
\left\|F^{\prime}\left(x_{0}\right)^{-1} F^{\prime \prime}(x)\right\| \leqslant \frac{2 \gamma}{\left(1-\gamma\left\|x-x_{0}\right\|\right)^{3}}, \\
x \in \mathbf{B}\left(x_{0}, r_{0}\right) .
\end{array}
$$

Then, for any $x \in \mathbf{B}\left(x_{0}, r_{0}\right)$, it follows from Lemma 1 that $F^{\prime}(x)^{-1}$ exists and the following inequality holds:

$$
\left\|F^{\prime}(x)^{-1} F^{\prime}\left(x_{0}\right)\right\| \leqslant \frac{\left(1-\gamma\left\|x-x_{0}\right\|\right)^{2}}{2\left(1-\gamma\left\|x-x_{0}\right\|\right)^{2}-1} .
$$

Below we list two useful lemmas.

Recall that the divided difference operator $[y, x ; F]$ is defined by (5). The following lemma gives the expressions of some desired estimates in the proof of Lemma 5.

Lemma 4. Let $x \in \mathbf{B}\left(x_{0}, r_{0}\right)$. Define

$$
y:=x-F^{\prime}(x)^{-1} F(x), \quad \bar{x}:=x-[y, x ; F]^{-1} F(x) .
$$

Then the following formulas hold:

(i) $[y, x ; F]-F^{\prime}\left(x_{0}\right)=\int_{0}^{1} \int_{0}^{1} F^{\prime \prime}\left(x_{0}+s\left[\left(x-x_{0}\right)+t(y-\right.\right.$ $x)])\left[\left(x-x_{0}\right)+t(y-x)\right] \mathrm{d} s \mathrm{~d} t$.

(ii) $\bar{x}-y=-F^{\prime}(x)^{-1} \int_{0}^{1} \int_{0}^{1} F^{\prime \prime}(x+s t(y-x))(y-x)(\bar{x}-$ $x) t \mathrm{~d} s \mathrm{~d} t$.

(iii) $F(\bar{x})=\int_{0}^{1} \int_{0}^{1} F^{\prime \prime}(x+t(y-x)+s t(\bar{x}-y))(\bar{x}-x)(\bar{x}-$ y)t $\mathrm{d} s \mathrm{~d} t$.

Proof. For (i), we notice that

$$
\begin{aligned}
{[y, x ; F]-F^{\prime}\left(x_{0}\right)=} & \int_{0}^{1}\left[F^{\prime}(x+t(y-x))-F^{\prime}\left(x_{0}\right)\right] \mathrm{d} t \\
= & \int_{0}^{1} \int_{0}^{1} F^{\prime \prime}\left(x_{0}+s\left[\left(x-x_{0}\right)+t(y-x)\right]\right) \\
& \times\left[\left(x-x_{0}\right)+t(y-x)\right] \mathrm{d} s \mathrm{~d} t .
\end{aligned}
$$

As for (ii), one has

$$
\begin{aligned}
\bar{x}-y= & F^{\prime}(x)^{-1} F(x)-[y, x ; F]^{-1} F(x) \\
= & F^{\prime}(x)^{-1}\left[F^{\prime}(x)-\int_{0}^{1} F^{\prime}(x+t(y-x)) \mathrm{d} t\right] \\
& \times(\bar{x}-x) \\
= & -F^{\prime}(x)^{-1} \\
& \times \int_{0}^{1} \int_{0}^{1} F^{\prime \prime}(x+s t(y-x))(y-x)(\bar{x}-x) t \mathrm{~d} s \mathrm{~d} t .
\end{aligned}
$$

Similarly, we obtain

$$
\begin{aligned}
F(\bar{x})= & F(\bar{x})-F(x)-[y, x ; F](\bar{x}-x) \\
= & \int_{0}^{1} F^{\prime}(x+t(\bar{x}-x))(\bar{x}-x) \mathrm{d} t \\
& -\int_{0}^{1} F^{\prime}(x+t(y-x))(\bar{x}-x) \mathrm{d} t \\
= & \int_{0}^{1} \int_{0}^{1} F^{\prime \prime}(x+t(y-x)+s t(\bar{x}-y)) \\
& \times(\bar{x}-x)(\bar{x}-y) t \mathrm{~d} s \mathrm{~d} t .
\end{aligned}
$$

The proof is complete.

Lemma 5. Suppose that (14) holds. Then the sequence $\left\{x_{n}\right\}$ generated by (4) with the initial point $x_{0}$ is well defined and the following estimates hold for any natural number $n \geqslant 1$ :

(i) $\left\|y_{n-1}-x_{n-1}\right\| \leqslant s_{n-1}-t_{n-1},\left\|x_{n}-x_{n-1}\right\| \leqslant t_{n}-t_{n-1}, \| x_{n}-$ $y_{n-1} \| \leqslant t_{n}-s_{n-1}$.

(ii) $\left\|\left[y_{n-1}, x_{n-1} ; F\right]^{-1} F^{\prime}\left(x_{0}\right)\right\| \leqslant-\left(\left(s_{n-1}-t_{n-1}\right) /\left(h\left(s_{n-1}\right)-\right.\right.$ $\left.\left.h\left(t_{n-1}\right)\right)\right)$.

(iii) $\left\|F^{\prime}\left(x_{0}\right)^{-1} F\left(x_{n}\right)\right\| \leqslant h\left(t_{n}\right)\left(\left\|x_{n}-x_{n-1}\right\| /\left(t_{n}-t_{n-1}\right)\right)^{2}$ $\left(\left\|y_{n-1}-x_{n-1}\right\| /\left(s_{n-1}-t_{n-1}\right)\right)$.

Proof. For the case $n=1$ in (i), it is clear that $\left\|y_{0}-x_{0}\right\| \leqslant$ $\beta=s_{0}-t_{0}$. By Lemma 4 and (29), we have

$$
\begin{aligned}
& \left\|F^{\prime}\left(x_{0}\right)^{-1}\left(\left[y_{0}, x_{0} ; F\right]-F^{\prime}\left(x_{0}\right)\right)\right\| \\
& =\| F^{\prime}\left(x_{0}\right)^{-1} \\
& \quad \quad \quad \quad \int_{0}^{1} \int_{0}^{1} F^{\prime \prime}\left(x_{0}+s t\left(y_{0}-x_{0}\right)\right)\left(y_{0}-x_{0}\right) t \mathrm{~d} s \mathrm{~d} t \| \\
& \leqslant \int_{0}^{1} \int_{0}^{1} \frac{2 \gamma\left(s_{0}-t_{0}\right) t}{\left[1-\gamma\left(s_{0}-t_{0}\right) s t\right]^{3}} \mathrm{~d} s \mathrm{~d} t \\
& =\frac{h\left(s_{0}\right)-h\left(t_{0}\right)}{s_{0}-t_{0}}+1 .
\end{aligned}
$$

In view of the monotonicity of $h$, one has that $\left(h\left(s_{0}\right)-\right.$ $\left.h\left(t_{0}\right)\right) /\left(s_{0}-t_{0}\right)<0$. Hence, we get from Banach lemma that $\left[y_{0}, x_{0} ; F\right]^{-1}$ exists and satisfies

$$
\begin{aligned}
\left\|\left[y_{0}, x_{0} ; F\right]^{-1} F^{\prime}\left(x_{0}\right)\right\| & \\
& \leqslant \frac{1}{1-\left(\left(\left(h\left(s_{0}\right)-h\left(t_{0}\right)\right) /\left(s_{0}-t_{0}\right)\right)+1\right)} \\
& =-\frac{s_{0}-t_{0}}{h\left(s_{0}\right)-h\left(t_{0}\right)} .
\end{aligned}
$$


Combining (36) inequality with the definitions of $\left\{s_{n}\right\}$ and $\left\{t_{n}\right\}$ given in (13), one has

$$
\begin{aligned}
\left\|x_{1}-x_{0}\right\| & \leqslant\left\|\left[y_{0}, x_{0} ; F\right]^{-1} F^{\prime}\left(x_{0}\right)\right\|\left\|F^{\prime}\left(x_{0}\right)^{-1} F\left(x_{0}\right)\right\| \\
& \leqslant-\frac{s_{0}-t_{0}}{h\left(s_{0}\right)-h\left(t_{0}\right)} h\left(t_{0}\right) \\
& =t_{1}-t_{0} .
\end{aligned}
$$

As for the estimate $\left\|x_{1}-y_{0}\right\|$, by Lemma 4 , we have

$$
\begin{aligned}
x_{1}-y_{0}= & -F^{\prime}\left(x_{0}\right)^{-1} \\
& \quad \times \int_{0}^{1} \int_{0}^{1} F^{\prime \prime}\left(x_{0}+s t\left(y_{0}-x_{0}\right)\right) \\
& \quad \times\left(y_{0}-x_{0}\right)\left(x_{1}-x_{0}\right) t \mathrm{~d} s \mathrm{~d} t .
\end{aligned}
$$

This together with the obtained bounds $\left\|y_{0}-x_{0}\right\|, \| x_{1}$ $x_{0} \|$ and (29) yields that

$$
\begin{aligned}
\left\|x_{1}-y_{0}\right\| \leqslant & \int_{0}^{1} \int_{0}^{1} \frac{2 \gamma\left(s_{0}-t_{0}\right)\left(t_{1}-t_{0}\right)}{\left(1-\gamma\left(s_{0}-t_{0}\right) s t\right)^{3}} t \mathrm{~d} s \mathrm{~d} t \\
& \times\left(\frac{\left\|y_{0}-x_{0}\right\|}{s_{0}-t_{0}}\right)\left(\frac{\left\|x_{1}-x_{0}\right\|}{t_{1}-t_{0}}\right) \\
= & \left(t_{1}-s_{0}\right)\left(\frac{\left\|y_{0}-x_{0}\right\|}{s_{0}-t_{0}}\right)\left(\frac{\left\|x_{1}-x_{0}\right\|}{t_{1}-t_{0}}\right) .
\end{aligned}
$$

This implies that statement (i) holds for $n=1$.

Statement (ii) for the case $n=1$ is justified by (36). Below, we consider the case $n=1$ for (iii). First we have the following expression of $F\left(x_{1}\right)$ due to Lemma 4:

$$
\begin{gathered}
F\left(x_{1}\right)=\int_{0}^{1} \int_{0}^{1} F^{\prime \prime}\left(x_{0}+t\left(y_{0}-x_{0}\right)+s t\left(x_{1}-y_{0}\right)\right) \\
\times\left(x_{1}-x_{0}\right)\left(x_{1}-y_{0}\right) t \mathrm{~d} s \mathrm{~d} t
\end{gathered}
$$

from which we obtain that

$$
\begin{aligned}
&\left\|F^{\prime}\left(x_{0}\right)^{-1} F\left(x_{1}\right)\right\| \\
& \leqslant \int_{0}^{1} \int_{0}^{1} \frac{2 \gamma\left(t_{1}-t_{0}\right)\left(t_{1}-s_{0}\right) t}{\left[1-\gamma\left(t\left(s_{0}-t_{0}\right)+s t\left(t_{1}-s_{0}\right)\right)\right]^{3}} \mathrm{~d} s \mathrm{~d} t \\
& \quad \times\left(\frac{\left\|x_{1}-x_{0}\right\|}{t_{1}-t_{0}}\right)^{2}\left(\frac{\left\|y_{0}-x_{0}\right\|}{s_{0}-t_{0}}\right) \\
&= h\left(t_{1}\right)\left(\frac{\left\|x_{1}-x_{0}\right\|}{t_{1}-t_{0}}\right)^{2}\left(\frac{\left\|y_{0}-x_{0}\right\|}{s_{0}-t_{0}}\right) .
\end{aligned}
$$

Therefore statement (iii) holds for $n=1$.

Assume that statements (i)-(iii) are true for $n=k(\geqslant 1)$. Below, we will show that they also hold for $n=k+1$. First, by statement (i), we have

$$
\begin{aligned}
\left\|x_{k}-x_{0}\right\| & \leqslant \sum_{i=0}^{k-1}\left\|x_{i+1}-x_{i}\right\| \leqslant \sum_{i=0}^{k-1}\left(t_{i+1}-t_{i}\right) \\
& =t_{k}<t^{*}<r_{0} .
\end{aligned}
$$

Hence, $F^{\prime}\left(x_{k}\right)^{-1}$ exists by Lemma 1 .
Note that

$$
\left\|F^{\prime}\left(x_{0}\right)^{-1} F\left(x_{k}\right)\right\| \leqslant h\left(t_{k}\right)
$$

by the inductive hypotheses of (i) and (iii). Then it follows from (30) and (13) that

$$
\begin{aligned}
\left\|y_{k}-x_{k}\right\| & \leqslant\left\|F^{\prime}\left(x_{k}\right)^{-1} F^{\prime}\left(x_{0}\right)\right\|\left\|F^{\prime}\left(x_{0}\right)^{-1} F\left(x_{k}\right)\right\| \\
& \leqslant-\frac{h\left(t_{k}\right)}{h^{\prime}\left(t_{k}\right)}=s_{k}-t_{k} .
\end{aligned}
$$

Hence by (29), (44), Lemma 4, and the inductive hypothesis of (i), we have

$$
\begin{aligned}
& \left\|F^{\prime}\left(x_{0}\right)^{-1}\left(\left[y_{k}, x_{k} ; F\right]-F^{\prime}\left(x_{0}\right)\right)\right\| \\
& =\| F^{\prime}\left(x_{0}\right)^{-1} \int_{0}^{1} \int_{0}^{1} F^{\prime \prime}\left(x_{0}+s\left[\left(x_{k}-x_{0}\right)+t\left(y_{k}-x_{k}\right)\right]\right) \\
& \quad \times\left[\left(x_{k}-x_{0}\right)+t\left(y_{k}-x_{k}\right)\right] \mathrm{d} s \mathrm{~d} t \| \\
& \leqslant \int_{0}^{1} \int_{0}^{1} \frac{2 \gamma}{\left[1-\gamma s\left(t_{k}+t\left(s_{k}-t_{k}\right)\right)\right]^{3}} \\
& =\int_{0}^{1}\left(\frac{1}{\left[1-\gamma\left(t_{k}+t\left(s_{k}-t_{k}\right)\right)\right]^{2}}-1\right) \mathrm{d} t \\
& =\frac{h\left(s_{k}\right)-h\left(t_{k}\right)}{s_{k}-t_{k}}+1<1 .
\end{aligned}
$$

It follows from Banach lemma that $\left[y_{k}, x_{k} ; F\right]^{-1} F^{\prime}\left(x_{0}\right)$ exists and satisfies

$$
\begin{aligned}
\left\|\left[y_{k}, x_{k} ; F\right]^{-1} F^{\prime}\left(x_{0}\right)\right\| \\
\quad \leqslant \frac{1}{1-\left(\left(\left(h\left(s_{k}\right)-h\left(t_{k}\right)\right) /\left(s_{k}-t_{k}\right)\right)+1\right)} \\
\quad=-\frac{s_{k}-t_{k}}{h\left(s_{k}\right)-h\left(t_{k}\right)} .
\end{aligned}
$$

Hence, (ii) holds for $n=\mathrm{k}+1$. 

has

Combining (46) with the inductive hypothesis (iii), one

$$
\begin{aligned}
\left\|x_{k+1}-x_{k}\right\| \leqslant & \left\|\left[y_{k}, x_{k} ; F\right]^{-1} F^{\prime}\left(x_{0}\right)\right\|\left\|F^{\prime}\left(x_{0}\right)^{-1} F\left(x_{k}\right)\right\| \\
\leqslant & -\left(\frac{h\left(s_{k}\right)-h\left(t_{k}\right)}{s_{k}-t_{k}}\right)^{-1} h\left(t_{k}\right) \\
& \times\left(\frac{\left\|x_{k}-x_{k-1}\right\|}{t_{k}-t_{k-1}}\right)^{2}\left(\frac{\left\|y_{k-1}-x_{k-1}\right\|}{s_{k-1}-t_{k-1}}\right) \\
= & \left(t_{k+1}-t_{k}\right) \\
& \times\left(\frac{\left\|x_{k}-x_{k-1}\right\|}{t_{k}-t_{k-1}}\right)^{2}\left(\frac{\left\|y_{k-1}-x_{k-1}\right\|}{s_{k-1}-t_{k-1}}\right),
\end{aligned}
$$

which implies that $\left\|x_{k+1}-x_{k}\right\| \leqslant t_{k+1}-t_{k}$.

On the other hand, by (29), (30), (44), and Lemma 4, we conclude that

$$
\begin{aligned}
& \left\|x_{k+1}-y_{k}\right\| \\
& \leqslant \int_{0}^{1} \int_{0}^{1}\left\|F^{\prime}\left(x_{k}\right)^{-1} F^{\prime \prime}\left(x_{k}+s t\left(y_{k}-x_{k}\right)\right)\right\| \\
& \times\left\|y_{k}-x_{k}\right\|\left\|x_{k+1}-x_{k}\right\| t \mathrm{~d} s \mathrm{~d} t \\
& \leqslant\left\|F^{\prime}\left(x_{k}\right)^{-1} F^{\prime}\left(x_{0}\right)\right\| \\
& \times \int_{0}^{1} \int_{0}^{1} \frac{2 \gamma\left\|y_{k}-x_{k}\right\|\left\|x_{k+1}-x_{k}\right\| t}{\left[1-\gamma\left(\left\|x_{k}-x_{0}\right\|+s t\left\|y_{k}-x_{k}\right\|\right)\right]^{3}} \mathrm{~d} s \mathrm{~d} t \\
& \leqslant \frac{\left(1-\gamma t_{k}\right)^{2}}{2\left(1-\gamma t_{k}\right)^{2}-1} \\
& \times \int_{0}^{1} \int_{0}^{1} \frac{2 \gamma\left(s_{k}-t_{k}\right)\left(t_{k+1}-t_{k}\right) t}{\left[1-\gamma\left(t_{k}+s t\left(s_{k}-t_{k}\right)\right)\right]^{3}} \mathrm{~d} s \mathrm{~d} t \\
& \times\left(\frac{\left\|y_{k}-x_{k}\right\|\left\|x_{k+1}-x_{k}\right\|}{\left(s_{k}-t_{k}\right)\left(t_{k+1}-t_{k}\right)}\right) \\
& =-\frac{1}{h^{\prime}\left(t_{k}\right)}\left(t_{k+1}-t_{k}\right)\left(\frac{h\left(s_{k}\right)-h\left(t_{k}\right)}{s_{k}-t_{k}}-h^{\prime}\left(t_{k}\right)\right) \\
& \times\left(\frac{\left\|y_{k}-x_{k}\right\|\left\|x_{k+1}-x_{k}\right\|}{\left(s_{k}-t_{k}\right)\left(t_{k+1}-t_{k}\right)}\right) \\
& =\left(t_{k+1}-s_{k}\right)\left(\frac{\left\|y_{k}-x_{k}\right\|\left\|x_{k+1}-x_{k}\right\|}{\left(s_{k}-t_{k}\right)\left(t_{k+1}-t_{k}\right)}\right) \text {, }
\end{aligned}
$$

which leads to $\left\|x_{k+1}-y_{k}\right\| \leqslant t_{k+1}-s_{k}$. Thus, (i) holds for $n=$ $k+1$.
Next, we will show that (iii) also holds for $n=k+1$. In fact, by using Lemma 4, (29), (44), and (48), we obtain

$$
\begin{aligned}
\| & F^{\prime}\left(x_{0}\right)^{-1} F\left(x_{k+1}\right) \| \\
\leqslant & \int_{0}^{1} \int_{0}^{1} \frac{2 \gamma\left(t_{k+1}-t_{k}\right)\left(t_{k+1}-s_{k}\right)}{\left[1-\gamma\left(t_{k}+t\left(s_{k}-t_{k}\right)+s t\left(t_{k+1}-s_{k}\right)\right)\right]^{3}} t \mathrm{~d} s \mathrm{~d} t \\
& \cdot\left(\frac{\left\|x_{k+1}-x_{k}\right\|}{t_{k+1}-t_{k}}\right)^{2}\left(\frac{\left\|y_{k}-x_{k}\right\|}{s_{k}-t_{k}}\right) \\
= & \left(h\left(t_{k+1}\right)-h\left(t_{k}\right)-\frac{t_{k+1}-t_{k}}{s_{k}-t_{k}}\left(h\left(s_{k}\right)-h\left(t_{k}\right)\right)\right) \\
& \cdot\left(\frac{\left\|x_{k+1}-x_{k}\right\|}{t_{k+1}-t_{k}}\right)^{2}\left(\frac{\left\|y_{k}-x_{k}\right\|}{s_{k}-t_{k}}\right) \\
\leqslant & h\left(t_{k+1}\right)\left(\frac{\left\|x_{k+1}-x_{k}\right\|}{t_{k+1}-t_{k}}\right)^{2}\left(\frac{\left\|y_{k}-x_{k}\right\|}{s_{k}-t_{k}}\right) .
\end{aligned}
$$

Therefore statement (iii) holds for $n=k+1$. Hence (i)-(iii) hold for all $n \geqslant 0$. Furthermore, by statement (i), one has, for any $n \geqslant 0,\left\|x_{n}-x_{0}\right\| \leqslant t_{n}<t^{*}<r_{0}$. Thus by Lemma 1 we know that $F^{\prime}\left(x_{n}\right)^{-1}$ exists for each $n \geqslant 1$; that is, $\left\{x_{n}\right\}$ is well defined. The proof is complete.

Recall that $\left\{s_{n}\right\}$ and $\left\{t_{n}\right\}$ are defined in (13). Based on the preceding useful lemmas, we are now ready to prove a Smaletype semilocal convergence theorem for Newton-Steffensen's method (4) under $\gamma$-condition.

Theorem 6. Suppose that (14) holds. Then the sequence $\left\{x_{n}\right\}$ generated by (4) with the initial point $x_{0}$ is well defined and converges to a solution $x^{*} \in \overline{\mathbf{B}\left(x_{0}, t^{*}\right)}$ of (1) with Q-cubic rate, and this solution $x^{*}$ is unique in $\overline{\mathbf{B}\left(x_{0}, r\right)}$, where $t^{*} \leqslant r<t^{* *}$. Moreover, the following error bounds

$$
\left\|x^{*}-x_{n}\right\| \leqslant\left(t^{*}-t_{n}\right)\left(\frac{\left\|x^{*}-x_{m}\right\|}{t^{*}-t_{m}}\right)^{3^{n-m}} \quad \forall n \geqslant m \geqslant 0
$$

are valid, where $t^{*}$ and $t^{* *}$ are defined in Lemma 2.

Proof. The uniqueness ball can be obtained by Theorem 5.2 in [19]. It follows from Lemma 1 that $\left\{x_{n}\right\}$ is well defined. In addition, from Lemmas 3 and 5 (i), we can see that $\left\{x_{n}\right\}$ is convergent to a limit, say $x^{*}$. Below, we show that $x^{*}$ is a solution of (1). It follows from Lemma 5 (iii) that

$$
\left\|F^{\prime}\left(x_{0}\right)^{-1} F\left(x_{n}\right)\right\| \leqslant h\left(t_{n}\right) \quad \forall n \geqslant 0 .
$$

Letting $n \rightarrow \infty$ in the preceding relation gives that the limit $x^{*}$ is a solution of (1). Moreover, we have

$$
\left\|x^{*}-x_{n}\right\| \leqslant t^{*}-t_{n}
$$


Next, we verify that estimate (62) is true. By (29) and Lemma 5, one has

$$
\begin{aligned}
& \left\|x^{*}-y_{n}\right\| \\
& =\| F^{\prime}\left(x_{n}\right)^{-1} \\
& \quad \times\left[\int_{0}^{1} F^{\prime}\left(x_{n}\right)\left(x^{*}-x_{n}\right) \mathrm{d} t+F\left(x_{n}\right)-F\left(x^{*}\right)\right] \| \\
& =\| F^{\prime}\left(x_{n}\right)^{-1} \\
& \quad \times \int_{0}^{1} \int_{0}^{1} F^{\prime \prime}\left(x_{n}+s t\left(x^{*}-x_{n}\right)\right)\left(x^{*}-x_{n}\right)^{2} t \mathrm{~d} s \mathrm{~d} t \| \\
& \leqslant\left\|F^{\prime}\left(x_{n}\right)^{-1} F^{\prime}\left(x_{0}\right)\right\| \\
& \quad \times \int_{0}^{1} \int_{0}^{1} F^{\prime}\left(x_{0}\right)^{-1} F^{\prime \prime}\left(x_{n}+s t\left(x^{*}-x_{n}\right)\right) \\
& \leqslant \frac{\left(1-\gamma\left\|x_{n}-x_{0}\right\|\right)^{2}}{2\left(1-\gamma\left\|x_{n}-x_{0}\right\|\right)^{2}-1} \\
& \quad \times \int_{0}^{1} \int_{0}^{1} \frac{2 \gamma\left(t^{*}-t_{n}\right)^{2} t}{\left[1-\gamma\left(\left\|x_{n}-x_{0}+s t\left(x^{*}-x_{n}\right)\right\|\right)\right]^{3}} \mathrm{~d} s \mathrm{~d} t \\
& \leqslant\left(t^{*}-s_{n}\right)\left(\frac{\left\|x^{*}-x_{n}\right\|}{t^{*}-t_{n}}\right)^{2} .
\end{aligned}
$$

In order to estimate $\left\|x_{n+1}-x^{*}\right\|$, we first notice that

$$
\begin{aligned}
& {\left[y_{n}, x_{n} ; F\right]\left(x_{n+1}-x^{*}\right)} \\
& \quad=\int_{0}^{1} \int_{0}^{1} F^{\prime \prime}\left(x_{n}+z_{n}^{s, t}\right)\left(x^{*}-y_{n}\right)\left(x^{*}-x_{n}\right) t \mathrm{~d} s \mathrm{~d} t,
\end{aligned}
$$

where $z_{n}^{s, t}=t\left(y_{n}-x_{n}\right)+s t\left(x^{*}-y_{n}\right)$. This together with Lemma 5(i), (29), (52), and (53) gives that

$$
\begin{aligned}
& \left\|F^{\prime}\left(x_{0}\right)^{-1}\left[y_{n}, x_{n} ; F\right]\left(x_{n+1}-x^{*}\right)\right\| \\
& \leqslant \int_{0}^{1} \int_{0}^{1} \frac{2 \gamma\left\|x^{*}-y_{n}\right\|\left\|x^{*}-x_{n}\right\| t}{\left[1-\gamma\left(\left\|x_{n}-x_{0}\right\|+t\left\|y_{n}-x_{n}\right\|+s t\left(x^{*}-y_{n}\right)\right)\right]^{3}} \mathrm{~d} s \mathrm{~d} t \\
& \leqslant \int_{0}^{1} \int_{0}^{1} \frac{2 \gamma\left(t^{*}-s_{n}\right)\left(t^{*}-t_{n}\right) t}{\left[1-\gamma\left(t_{n}+t\left(s_{n}-t_{n}\right)+s t\left(t^{*}-s_{n}\right)\right)\right]^{3}} \mathrm{~d} s \mathrm{~d} t \\
& \quad \times\left(\frac{\left\|x^{*}-x_{n}\right\|}{t^{*}-t_{n}}\right)^{3}
\end{aligned}
$$

$$
\begin{aligned}
& =\left(-h\left(t_{n}\right)-\frac{h\left(s_{n}\right)-h\left(t_{n}\right)}{s_{n}-t_{n}}\left(t^{*}-t_{n}\right)\right)\left(\frac{\left\|x^{*}-x_{n}\right\|}{t^{*}-t_{n}}\right)^{3} \\
& =\left(t_{n+1}-t^{*}\right)\left(\frac{h\left(s_{n}\right)-h\left(t_{n}\right)}{s_{n}-t_{n}}\right)\left(\frac{\left\|x^{*}-x_{n}\right\|}{t^{*}-t_{n}}\right)^{3} .
\end{aligned}
$$

Combining the above inequality with (46), we have

$$
\begin{aligned}
\left\|x_{n+1}-x^{*}\right\| \leqslant & \left\|\left[y_{n}, x_{n} ; F\right]^{-1} F^{\prime}\left(x_{0}\right)\right\| \\
& \times\left\|F^{\prime}\left(x_{0}\right)^{-1}\left[y_{n}, x_{n} ; F\right]\left(x_{n+1}-x^{*}\right)\right\| \\
\leqslant & -\left(\frac{h\left(s_{n}\right)-h\left(t_{n}\right)}{s_{n}-t_{n}}\right)^{-1}\left(t_{n+1}-t^{*}\right) \\
& \times\left(\frac{h\left(s_{n}\right)-h\left(t_{n}\right)}{s_{n}-t_{n}}\right)\left(\frac{\left\|x^{*}-x_{n}\right\|}{t^{*}-t_{n}}\right)^{3} \\
\leqslant & \left(t^{*}-t_{n+1}\right)\left(\frac{\left\|x^{*}-x_{n}\right\|}{t^{*}-t_{n}}\right)^{3} .
\end{aligned}
$$

Therefore, the error estimate (62) follows. Also, from the previous inequality, we know that the convergence rate of $\left\{x_{n}\right\}$ to $x^{*}$ is Q-cubic. This completes the proof.

One typical and important class of examples satisfying $\gamma$ condition is the one of analytic functions. Smale [5] studied the convergence and error estimation of Newton's method (2) under the hypotheses that $F$ is analytic and satisfies

$$
\left\|F^{\prime}(\bar{x})^{-1} F^{(n)}(\bar{x})\right\| \leqslant n ! \gamma^{n-1}, \quad n \geqslant 2,
$$

where $\bar{x}$ is a fixed point in $D$ and $\gamma$ is defined by

$$
\gamma:=\sup _{n>1}\left\|\frac{F^{\prime}(\bar{x})^{-1} F^{(n)}(\bar{x})}{n !}\right\|^{1 /(n-1)} .
$$

The following lemma taken from [20] shows that an analytic operator satisfies $\gamma$-condition.

Lemma 7. Let $r_{0}$ and $\gamma$ be defined by (7) and (86), respectively. Suppose that $F$ is analytic and satisfies (85). Then $F$ satisfies $\gamma$-condition

$$
\left\|F^{\prime}(\bar{x})^{-1} F^{\prime \prime}(x)\right\| \leqslant \frac{2 \gamma}{(1-\gamma\|x-\bar{x}\|)^{3}}
$$

on $B\left(\bar{x}, r_{0}\right)$.

According to this lemma, we can conclude that the semilocal results obtained in Theorem 6 also hold when $F$ is an analytic operator. 
Corollary 8. Suppose that (14) holds. Suppose that F is analytic and satisfies

$$
\left\|F^{\prime}\left(x_{0}\right)^{-1} F^{(n)}\left(x_{0}\right)\right\| \leqslant n ! \gamma^{n-1}, \quad n \geqslant 2,
$$

where $\gamma$ is defined by

$$
\gamma:=\sup _{n>1}\left\|\frac{F^{\prime}\left(x_{0}\right)^{-1} F^{(n)}\left(x_{0}\right)}{n !}\right\|^{1 /(n-1)} .
$$

Then the sequence $\left\{x_{n}\right\}$ generated by (4) with the initial point $x_{0}$ is well defined and converges to a solution $x^{*} \epsilon$ $\overline{\mathbf{B}\left(x_{0}, t^{*}\right)}$ of (1) with Q-cubic rate and this solution $x^{*}$ is unique in $\overline{\mathbf{B}\left(x_{0}, r\right)}$, where $t^{*} \leqslant r<t^{* *}$. Moreover, the following error bounds

$$
\left\|x^{*}-x_{n}\right\| \leqslant\left(t^{*}-t_{n}\right)\left(\frac{\left\|x^{*}-x_{m}\right\|}{t^{*}-t_{m}}\right)^{3^{n-m}} \quad \forall n \geqslant m \geqslant 0
$$

are valid, where $t^{*}$ and $t^{* *}$ are defined in Lemma 2 .

\section{Convergence Ball}

Now we begin to study the local convergence properties for Newton-Steffensen's method (4) under $\gamma$-condition. Recall that $r_{0}$ is defined by (7). Throughout this section, suppose that $x^{*} \in D$ such that $F\left(x^{*}\right)=0, \mathbf{B}\left(x^{*}, r_{0}\right) \subset D$, and the inverse $F^{\prime}\left(x^{*}\right)^{-1}$ exists. Moreover, we assume that $F$ satisfies the $\gamma$-condition on $\mathbf{B}\left(x^{*}, r_{0}\right)$; that is, the following relation holds:

$$
\left\|F^{\prime}\left(x^{*}\right)^{-1} F^{\prime \prime}(x)\right\| \leqslant \frac{2 \gamma}{\left(1-\gamma\left\|x-x^{*}\right\|\right)^{3}}, \quad x \in \mathbf{B}\left(x^{*}, r_{0}\right) .
$$

Then, for any $x \in \mathbf{B}\left(x^{*}, r_{0}\right)$, it follows from Lemma 1 that

$$
\left\|F^{\prime}(x)^{-1} F^{\prime}\left(x^{*}\right)\right\| \leqslant \frac{\left(1-\gamma\left\|x-x^{*}\right\|\right)^{2}}{2\left(1-\gamma\left\|x-x^{*}\right\|\right)^{2}-1} .
$$

Let

$$
r_{1}:=\frac{(5-\sqrt{17})}{(4 \gamma)} .
$$

Define function $G$ as follows:

$$
G(t)=\frac{\gamma t}{1-4 \gamma t+2 \gamma^{2} t^{2}}, \quad t \in\left(0, r_{1}\right) .
$$

It is clear that $r_{1} \in\left(0, r_{0}\right)$ and that $G\left(r_{1}\right)=1$. Moreover, $G$ increases monotonically in $\left(0, r_{1}\right)$.

Theorem 9. Let $r_{1}$ be defined in (65). Then, for any $x_{0} \in B\left(x^{*}, r_{1}\right)$, the sequence $\left\{x_{n}\right\}$ generated by (4) converges to $x^{*}$ and satisfies that

$$
\left\|x_{n}-x^{*}\right\| \leqslant q^{3^{n}-1}\left\|x_{0}-x^{*}\right\|, \quad n=0,1, \ldots,
$$

where

$$
q=G\left(t_{0}\right)<1, \quad t_{0}=\left\|x_{0}-x^{*}\right\| .
$$

Proof. For $n=0,1, \ldots$, we write $t_{n}=\left\|x_{n}-x^{*}\right\|$. It is sufficient to show that

$$
t_{n+1} \leqslant t_{n}, \quad\left\|x_{n+1}-x^{*}\right\| \leqslant\left(\frac{G\left(t_{n}\right)}{t_{n}}\right)^{2}\left\|x_{n}-x^{*}\right\|^{3},
$$

$$
n=0,1, \ldots
$$

In fact, by noticing the monotonicity of $G / t$, we have

$$
\begin{aligned}
\left\|x_{n+1}-x^{*}\right\| & \leqslant\left(\frac{G\left(t_{n}\right)}{t_{n}}\right)^{2}\left\|x_{n}-x^{*}\right\|^{3} \\
& \leqslant\left(\frac{G\left(t_{0}\right)}{t_{0}}\right)^{2}\left\|x_{n}-x^{*}\right\|^{3} \\
& =\left(\frac{q}{t_{0}}\right)^{2}\left\|x_{n}-x^{*}\right\|^{3}, \quad n=0,1, \ldots
\end{aligned}
$$

From this we can easily establish (67) by mathematical induction.

We now prove (69). First we get the following expression of $x_{n+1}-x^{*}$ :

$$
\begin{aligned}
x_{n+1}- & x^{*} \\
= & {\left[y_{n}, x_{n} ; F\right]^{-1} } \\
& \times\left[\int_{0}^{1} F^{\prime}\left(x_{n}+t\left(y_{n}-x_{n}\right)\right)\left(x_{n}-x^{*}\right) \mathrm{d} t\right. \\
& \left.-\left(F\left(x_{n}\right)-F\left(x^{*}\right)\right)\right] \\
= & {\left[y_{n}, x_{n} ; F\right]^{-1} } \\
& \times \int_{0}^{1} \int_{0}^{1} F^{\prime \prime}\left(x^{*}+(1-t)\left(x_{n}-x^{*}\right)+s t\left(y_{n}-x^{*}\right)\right) \\
& \times\left(y_{n}-x^{*}\right)\left(x_{n}-x^{*}\right) t \mathrm{~d} s \mathrm{~d} t .
\end{aligned}
$$

Similarly, we also have

$$
\begin{aligned}
y_{n}-x^{*}= & x_{n}-F^{\prime}\left(x_{n}\right)^{-1} F^{\prime}\left(x_{n}\right)-x^{*} \\
= & F^{\prime}\left(x_{n}\right)^{-1} \\
& \times\left[F\left(x^{*}\right)-F\left(x_{n}\right)+F^{\prime}\left(x_{n}\right)\left(x_{n}-x^{*}\right)\right] \\
= & F^{\prime}\left(x_{n}\right)^{-1} \\
& \times \int_{0}^{1}\left[F^{\prime}\left(x_{n}+t\left(x^{*}-x_{n}\right)\right)-F^{\prime}\left(x_{n}\right)\right] \\
& \times\left(x^{*}-x_{n}\right) \mathrm{d} t \\
= & F^{\prime}\left(x_{n}\right)^{-1} \\
& \times \int_{0}^{1} \int_{0}^{1} F^{\prime \prime}\left(x_{n}+s t\left(x^{*}-x_{n}\right)\right)\left(x^{*}-x_{n}\right)^{2} t \mathrm{~d} s \mathrm{~d} t .
\end{aligned}
$$


This together with (63) and (64) yields

$$
\begin{aligned}
& \left\|y_{n}-x^{*}\right\| \leqslant\left\|F^{\prime}\left(x_{n}\right)^{-1} F^{\prime}\left(x^{*}\right)\right\| \\
& \times\left\|\int_{0}^{1} \int_{0}^{1} F^{\prime}\left(x^{*}\right)^{-1} F^{\prime \prime}\left(x_{n}+s t\left(x^{*}-x_{n}\right)\right) t d s d t\right\| \\
& \times\left\|x^{*}-x_{n}\right\|^{2} \\
& \leqslant\left(\frac{1}{\left(1-\gamma\left\|x^{*}-x_{n}\right\|\right)^{2}}-2\right) \\
& \times \int_{0}^{1} \int_{0}^{1} \frac{2 \gamma}{\left[1-\gamma\left\|x_{n}+s t\left(x^{*}-x_{n}\right)-x^{*}\right\|\right]^{3}} \mathrm{~d} s \mathrm{~d} t \\
& \times\left\|x^{*}-x_{n}\right\|^{2} \\
& =\left(\frac{1}{\left(1-\gamma t_{n}\right)^{2}}-2\right) \\
& \times \int_{0}^{1} \int_{0}^{1} \frac{2 \gamma}{\left[1-\gamma(1-s t) t_{n}\right]^{3}} \mathrm{~d} s \mathrm{~d} t \\
& \times\left\|x^{*}-x_{n}\right\|^{2} \\
& =\frac{\gamma}{1-4 \gamma t_{n}+2 \gamma^{2} t_{n}^{2}}\left\|x^{*}-x_{n}\right\|^{2} \\
& =\left(\frac{G\left(t_{n}\right)}{t_{n}}\right)\left\|x^{*}-x_{n}\right\|^{2} \text {. }
\end{aligned}
$$

On the other hand, we notice that

$$
\begin{aligned}
& F^{\prime}\left(x^{*}\right)^{-1}\left(\left[y_{n}, x_{n} ; F\right]-F^{\prime}\left(x^{*}\right)\right) \\
& =F^{\prime}\left(x^{*}\right)^{-1} \\
& \times \int_{0}^{1} \int_{0}^{1} F^{\prime \prime}\left(x^{*}+s(1-t)\left(x_{n}-x^{*}\right)\right. \\
& \left.\quad+s t\left(y_{n}-x^{*}\right)\right) \\
& \quad \times\left[(1-t)\left(x_{n}-x^{*}\right)+t\left(y_{n}-x^{*}\right)\right] \mathrm{d} s \mathrm{~d} t .
\end{aligned}
$$

It follows from (63) that

$$
\begin{aligned}
& \left\|F^{\prime}\left(x^{*}\right)^{-1}\left(\left[y_{n}, x_{n} ; F\right]-F^{\prime}\left(x^{*}\right)\right)\right\| \\
& \leqslant \int_{0}^{1} \int_{0}^{1} \frac{2 \gamma\left[(1-t)\left\|x_{n}-x^{*}\right\|+t\left\|y_{n}-x^{*}\right\|\right]}{\left[1-\gamma\left(s(1-t)\left\|x_{n}-x^{*}\right\|+s t\left\|y_{n}-x^{*}\right\|\right)\right]^{3}} \mathrm{~d} s \mathrm{~d} t .
\end{aligned}
$$

For the case $n=0$, by (88) and (73), we get

$$
\left\|y_{0}-x^{*}\right\| \leqslant G\left(t_{0}\right)\left\|x^{*}-x_{0}\right\| \leqslant\left\|x^{*}-x_{0}\right\| .
$$

Combining Lemma 2 with (75) and (76), we obtain

$$
\begin{aligned}
& \left\|F^{\prime}\left(x^{*}\right)^{-1}\left(\left[y_{0}, x_{0} ; F\right]-F^{\prime}\left(x^{*}\right)\right)\right\| \\
& \leqslant \int_{0}^{1} \int_{0}^{1} \frac{2 \gamma\left[(1-t)\left\|x_{0}-x^{*}\right\|+t\left\|y_{0}-x^{*}\right\|\right]}{\left[1-\gamma\left(s(1-t)\left\|x_{0}-x^{*}\right\|+s t\left\|y_{0}-x^{*}\right\|\right)\right]^{3}} \mathrm{~d} s \mathrm{~d} t \\
& \leqslant \int_{0}^{1} \int_{0}^{1} \frac{2 \gamma\left[(1-t) t_{0}+t t_{0}\right]}{\left[1-\gamma\left(s(1-t) t_{0}+s t t_{0}\right)\right]^{3}} \mathrm{~d} s \mathrm{~d} t \\
& =\frac{1}{\left(1-\gamma t_{0}\right)^{2}}-1<1 .
\end{aligned}
$$

It follows from Banach lemma that

$$
\begin{aligned}
\left\|\left[y_{0}, x_{0} ; F\right]^{-1} F^{\prime}\left(x^{*}\right)\right\| & \leqslant \frac{1}{1-\left(\left(1 /\left(1-\gamma t_{0}\right)^{2}\right)-1\right)} \\
& =\frac{\left(1-\gamma t_{0}\right)^{2}}{2\left(1-\gamma t_{0}\right)^{2}-1} .
\end{aligned}
$$

This together with (63), (71) and (76) yields

$$
\begin{aligned}
& \left\|x_{1}-x^{*}\right\| \\
& \leqslant\left\|\left[y_{0}, x_{0} ; F\right]^{-1} F^{\prime}\left(x^{*}\right)\right\| \\
& \times \| F^{\prime}\left(x^{*}\right)^{-1} \\
& \times \int_{0}^{1} \int_{0}^{1} F^{\prime \prime}\left(x^{*}+(1-t)\left(x_{0}-x^{*}\right)\right. \\
& \left.+s t\left(y_{0}-x^{*}\right)\right) t \mathrm{~d} s \mathrm{~d} t \| \\
& \times\left\|y_{0}-x^{*}\right\|\left\|x_{0}-x^{*}\right\| \\
& \leqslant \frac{\left(1-\gamma t_{0}\right)^{2}}{2\left(1-\gamma t_{0}\right)^{2}-1} \\
& \times \int_{0}^{1} \int_{0}^{1} \frac{2 \gamma}{\left[1-\gamma\left((1-t) t_{0}+s t t_{0}\right)\right]^{3}} t \mathrm{~d} s \mathrm{~d} t \\
& \times G\left(t_{0}\right)\left\|x^{*}-x_{0}\right\|^{3} \\
& =\frac{\left(1-\gamma t_{0}\right)^{2}}{2\left(1-\gamma t_{0}\right)^{2}-1} \frac{1}{t_{0}}\left(\frac{1}{\left(1-\gamma t_{0}\right)^{2}}-\frac{1}{\left(1-\gamma t_{0}\right)}\right) \\
& \times G\left(t_{0}\right)\left\|x^{*}-x_{0}\right\|^{3} \\
& =\left(\frac{G\left(t_{0}\right)}{t_{0}}\right)^{2}\left\|x^{*}-x_{0}\right\|^{3} \text {. }
\end{aligned}
$$

Hence (69) holds for $n=0$.

Now assume that the inequalities in (69) hold for up to some $n \geqslant 1$. Then by (73), one has

$$
\left\|y_{n+1}-x^{*}\right\|=G\left(t_{n+1}\right) t_{n+1} \leqslant G\left(t_{0}\right) t_{n+1} \leqslant t_{n+1} .
$$


Thus (75) can be further reduced to

$$
\begin{aligned}
& \left\|F^{\prime}\left(x^{*}\right)^{-1}\left(\left[y_{n+1}, x_{n+1} ; F\right]-F^{\prime}\left(x^{*}\right)\right)\right\| \\
& \quad \leqslant \int_{0}^{1} \int_{0}^{1} \frac{2 \gamma\left[(1-t) t_{n+1}+t t_{n+1}\right]}{\left[1-\gamma\left(s(1-t) t_{n+1}+s t t_{n+1}\right)\right]^{3}} \mathrm{~d} s \mathrm{~d} t \\
& \quad=\frac{1}{\left(1-\gamma t_{n+1}\right)^{2}}-1<1 .
\end{aligned}
$$

Using Banach lemma again, one has

$$
\left\|\left[y_{n+1}, x_{n+1} ; F\right]^{-1} F^{\prime}\left(x^{*}\right)\right\| \leqslant \frac{\left(1-\gamma t_{n+1}\right)^{2}}{2\left(1-\gamma t_{n+1}\right)^{2}-1} .
$$

This together with (63), (71), and (73) yields

$$
\begin{aligned}
& \left\|x_{n+2}-x^{*}\right\| \\
& \leqslant\left\|\left[y_{n+1}, x_{n+1} ; F\right]^{-1} F^{\prime}\left(x^{*}\right)\right\| \\
& \times \| F^{\prime}\left(x^{*}\right)^{-1} \\
& \cdot \int_{0}^{1} \int_{0}^{1} F^{\prime \prime}\left(x^{*}+(1-t)\left(x_{n+1}-x^{*}\right)\right. \\
& \left.+s t\left(y_{n+1}-x^{*}\right)\right) t \mathrm{~d} s \mathrm{~d} t \| \\
& \times\left\|y_{n+1}-x^{*}\right\|\left\|x_{n+1}-x^{*}\right\| \\
& \leqslant \frac{\left(1-\gamma t_{n+1}\right)^{2}}{2\left(1-\gamma t_{n+1}\right)^{2}-1} \\
& \times \int_{0}^{1} \int_{0}^{1} \frac{2 \gamma}{\left[1-\gamma\left((1-t) t_{n+1}+s t t_{n+1}\right)\right]^{3}} t \mathrm{~d} s \mathrm{~d} t \\
& \times G\left(t_{n+1}\right)\left\|x^{*}-x_{0}\right\|^{3} \\
& =\frac{\left(1-\gamma t_{n+1}\right)^{2}}{2\left(1-\gamma t_{n+1}\right)^{2}-1} \\
& \times \frac{1}{t_{n+1}}\left(\frac{1}{\left(1-\gamma t_{n+1}\right)^{2}}-\frac{1}{\left(1-\gamma t_{n+1}\right)}\right) \\
& \times G\left(t_{n+1}\right)\left\|x^{*}-x_{0}\right\|^{3} \\
& =\left(\frac{G\left(t_{n+1}\right)}{t_{n+1}}\right)^{2}\left\|x^{*}-x_{n+1}\right\|^{3} \text {. }
\end{aligned}
$$

Thus

$$
t_{n+2} \leq G\left(t_{n+1}\right)^{2} t_{n+1} \leq G\left(t_{0}\right)^{2} t_{n+1} \leq t_{n+1} .
$$

This and (83) show that the inequalities in (69) hold for $n+$ 1 and hence they hold for each $n$. The proof is complete.

Applying Lemma 7 to the above theorem, we get the following corollary:
Corollary 10. Suppose that $F$ is analytic and satisfies

$$
\left\|F^{\prime}\left(x^{*}\right)^{-1} F^{(n)}\left(x^{*}\right)\right\| \leqslant n ! \gamma^{n-1}, \quad n \geqslant 2,
$$

where $\gamma$ is defined by

$$
\gamma:=\sup _{n>1}\left\|\frac{F^{\prime}\left(x^{*}\right)^{-1} F^{(n)}\left(x^{*}\right)}{n !}\right\|^{1 /(n-1)} .
$$

Let $r_{1}$ and $G$ be defined by (65) and (66), respectively. Then, for any $x_{0} \in B\left(x^{*}, r_{1}\right)$, the sequence $\left\{x_{n}\right\}$ generated by (4) converges to $x^{*}$ and satisfies that

$$
\left\|x_{n}-x^{*}\right\| \leqslant q^{3^{n}-1}\left\|x_{0}-x^{*}\right\|, \quad n=0,1, \ldots,
$$

where

$$
q=G\left(t_{0}\right)<1, \quad t_{0}=\left\|x_{0}-x^{*}\right\| .
$$

\section{Conflict of Interests}

The authors declare that there is no conflict of interests regarding the publication of this paper.

\section{Acknowledgment}

Xiubin Xu's work was supported in part by the National Natural Science Foundation of China (Grant no. 61170109).

\section{References}

[1] L. Blum, F. Cucker, M. Shub, and S. Smale, Complexity and Real Computation, Springer, New York, NY, USA, 1998.

[2] J.-S. He, J.-H. Wang, and C. Li, "Newton's method for underdetermined systems of equations under the $\gamma$-condition," Numerical Functional Analysis and Optimization, vol. 28, no. 5-6, pp. 663-679, 2007

[3] C. Li, N. Hu, and J. Wang, "Convergence behavior of GaussNewton's method and extensions of the Smale point estimate theory," Journal of Complexity, vol. 26, no. 3, pp. 268-295, 2010.

[4] S. Smale, "The fundamental theorem of algebra and complexity theory," Bulletin of the American Mathematical Society, vol. 4, no. 1, pp. 1-36, 1981.

[5] S. Smale, "Newton's method estimates from data at one point," in The Merging of Disciplines: New Directions in Pure, Applied, and Computational Mathematics, pp. 185-196, Springer, New York, NY, USA, 1986.

[6] S. Smale, "Complexity theory and numerical analysis," Acta Numerica, vol. 6, pp. 523-551, 1997.

[7] X. Wang, "Convergence on the iteration of Halley family in weak conditions," Chinese Science Bulletin, vol. 42, no. 7, pp. 552-555, 1997.

[8] X. H. Wang and D. F. Han, "Criterion $\alpha$ and Newton's method under weak conditions," Mathematica Numerica Sinica, vol. 19, no. 1, pp. 96-105, 1997.

[9] V. Candela and A. Marquina, "Recurrence relations for rational cubic methods-I. The Halley method," Computing, vol. 44, no. 2, pp. 169-184, 1990. 
[10] V. Candela and A. Marquina, "Recurrence relations for rational cubic methods-II. The Chebyshev method," Computing, vol. 45, no. 4, pp. 355-367, 1990.

[11] M. A. Hernández and M. A. Salanova, "A family of ChebyshevHalley type methods," International Journal of Computer Mathematics, vol. 47, pp. 59-63, 1993.

[12] H. Wang, C. Li, and X. Wang, "On relationship between convergence ball of Euler iteration in Banach spaces and its dynamical behavior on Riemann spheres," Science in China. Mathematics, vol. 46, no. 3, pp. 376-382, 2003.

[13] X. Wang and C. Li, "On the united theory of the family of Euler-Halley type methods with cubical convergence in Banach spaces," Journal of Computational Mathematics, vol. 21, no. 2, pp. 195-200, 2003.

[14] X. Xu and Y. Ling, "Semilocal convergence for Halley's method under weak Lipschitz condition," Applied Mathematics and Computation, vol. 215, no. 8, pp. 3057-3067, 2009.

[15] X. Xu and Y. Ling, "Semilocal convergence for a family of Chebyshev-Halley like iterations under a mild differentiability condition," Journal of Applied Mathematics and Computing, vol. 40, no. 1-2, pp. 627-647, 2012.

[16] X. Ye and C. Li, "Convergence of the family of the deformed Euler-Halley iterations under the Hölder condition of the second derivative," Journal of Computational and Applied Mathematics, vol. 194, no. 2, pp. 294-308, 2006.

[17] J. R. Sharma, "A composite third order Newton-Steffensen method for solving nonlinear equations," Applied Mathematics and Computation, vol. 169, no. 1, pp. 242-246, 2005.

[18] X. H. Wang and D. F. Han, "On dominating sequence method in the point estimate and Smale theorem," Science in China. Mathematics, vol. 33, no. 2, pp. 135-144, 1990.

[19] X. Wang, "Convergence of Newton's method and inverse function theorem in Banach space," Mathematics of Computation, vol. 68, no. 225, pp. 169-186, 1999.

[20] X. H. Wang, D. F. Han, and F. Y. Sun, "Point estimates for some deformation Newton iterations," Mathematica Numerica Sinica, vol. 12, no. 2, pp. 145-156, 1990. 


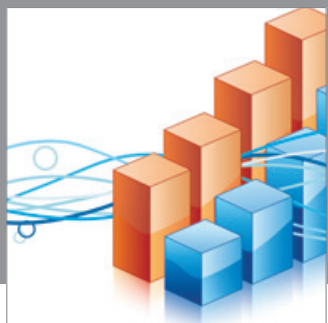

Advances in

Operations Research

mansans

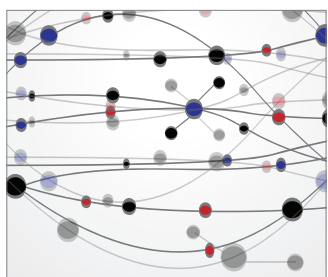

The Scientific World Journal
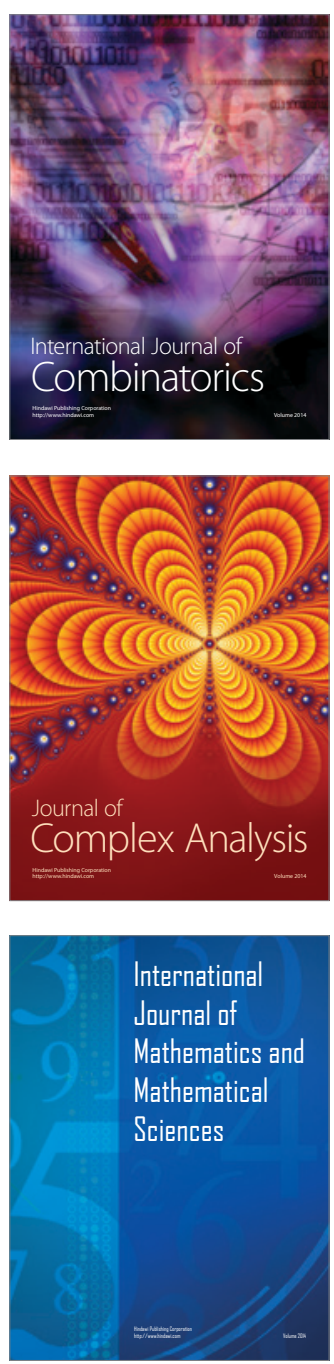
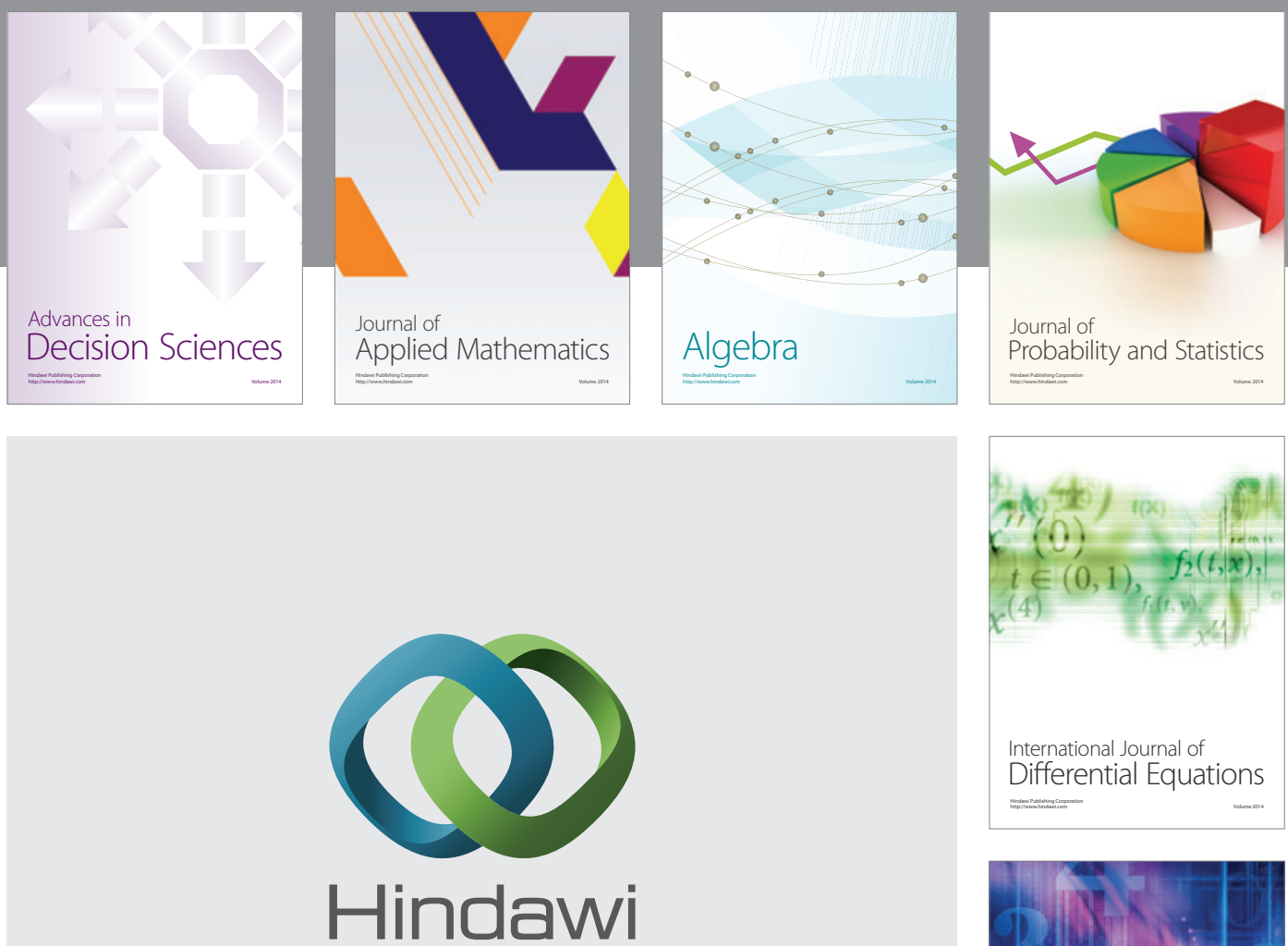

Submit your manuscripts at http://www.hindawi.com
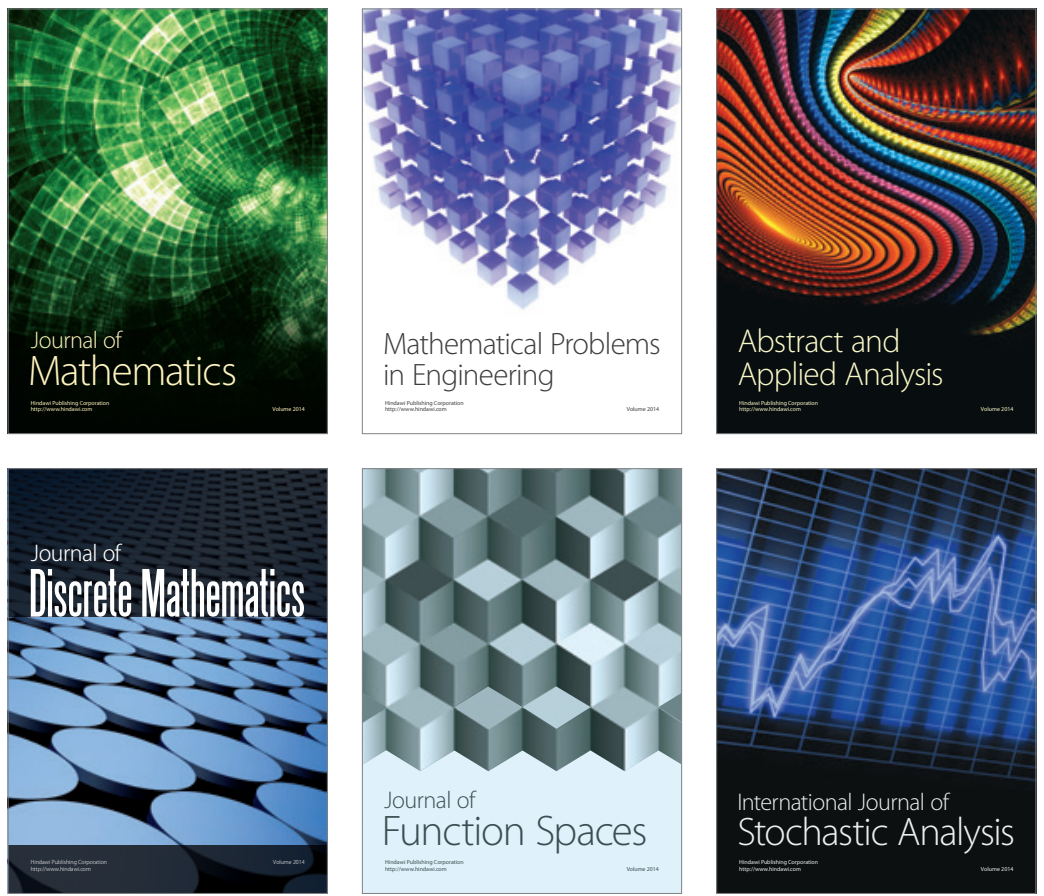

Journal of

Function Spaces

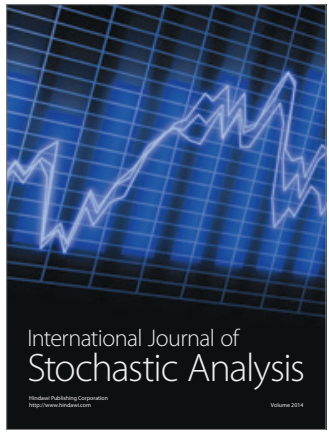

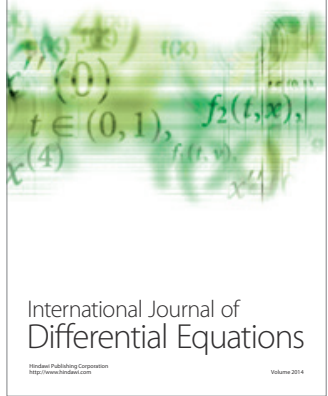
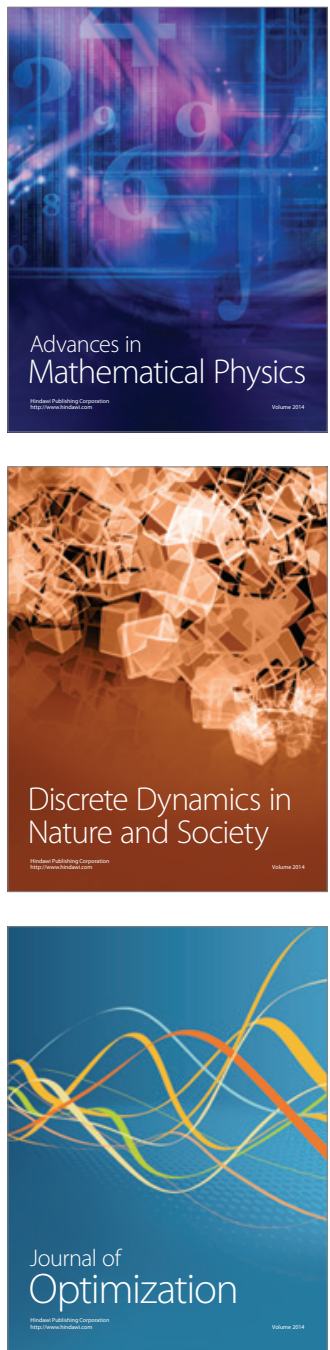